\title{
Physiology of the Basal Ganglia: An Overview
}

\author{
Robert G. Lee
}

ABSTRACT: The major anatomical connections of the basal ganglia are reviewed, emphasizing the inputs to the striatum and efferent projections from the major output nuclei, the internal segment of globus pallidus and the pars reticulata of substantia nigra. The results from lesioning experiments, electrical stimulation, and chronic recording of single neuron activity have provided a wealth of data concerning the physiology of the basal ganglia. Although the deficits resulting from disease of the basal ganglia are well recognized, the specific role which these structures play in the control of normal movements remains speculative.

RÉSUMÉ: Nous revisons les principales connections anatomiques des noyaux gris centraux, insistant particulièrement sur les entrées au striatum et les projections efférentes des principaux noyaux de sortie: le segment interne du globus pallidus et la pars reticulata de la substance noire. Les résultats d'expériences de lésions, de stimulations et d'enregistrements chroniques monocellulaires, produisent une pléthore de données sur la physiologie de ces noyaux. Malgré qu'on connaisse bien les déficits secondaires aux maladies de ces noyaux, on ne connait pas encore bien le rôle spécifique de ces structures dans le contrôle des mouvements normaux.

Can. J. Neurol. Sci. 1984; 11:124-128

The basal ganglia have attracted the interest of some of the best known figures in clinical neurology such as Kinnier Wilson, Purdon Martin and Denny-Brown. As a result of careful clinical observations by these individuals and many others, we have some appreciation of the wide variety of neurological deficits which result from disease processes affecting the basal ganglia. Disorders of the basal ganglia are characteristically associated with disturbances of posture and movement, and it has seemed a logical step to assume that these structures play an important role in the control of normal motor activity. However, despite the fact that the basal ganglia have been the focus of intensive anatomical and physiological investigation for the past two decades, the exact nature of this role remains unclear.

No attempt will be made to touch on all aspects of basal ganglia physiology in this brief survey. The topic has been covered in depth in several recent reviews (Delong and Georgopoulos, 1981; Kitai, 1981; Marsden, 1982; Penney and Young, 1983). The aim here will be to provide an overview of the normal function of the basal ganglia to serve as a basis for consideration of the pathophysiology of Parkinson's disease (see papers by Marsden and Tatton in in this volume).

\section{Anatomical Considerations}

It is impossible to discuss function without first considering structure and we shall begin by reviewing some of the functionally important anatomical connections in the basal ganglia. The anatomy of the basal ganglia is complex and most of the individual nuclei are interconnected to some degree with all the other nuclei in the basal ganglia. However, there are several general principles of anatomical organization which may provide clues concerning function. First, the majority of the inputs to the basal ganglia originate from the cerebral cortex. In contrast to the cerebellum, the basal ganglia receive no direct sensory input from the periphery. Secondly, a well developed somatotopic organization is maintained at all levels as information passes through the basal ganglia. Thirdly, most of the output from the basal ganglia is directed via the thalamus back to the cerebral cortex. There are no known output projections extending below the level of the mesencephalon. Therefore, the basal ganglia have no direct influence on the activity of spinal or brainstem motoneurons. Their main action is through "intermediaries", particularly various areas of the cerebral cortex.

A simplified schematic wiring diagram is presented in Figure 1. Anatomical purists may be disturbed by the fact that this illustration only remotely resembles the normal appearance of the basal ganglia. To emphasize functional connections and the normal flow of information through the basal ganglia, considerable liberty has been taken in the placement of various structures and a number of known anatomical connections have been omitted. The illustration is arranged so that the major inputs to the basal ganglia appear on the left and output connections appear on the right. As a result, components of some structures such as the substantia nigra and globus pallidus have become widely separated in the illustration even though they are normally in close anatomical proximity.

The striatum is the major receiving area for afferent input to the basal ganglia. The majority of the inputs of the striatum originate from various regions of the cerebral cortex. These projections have an exitatory effect on striatal neurons and utilize glutamate as a neurotransmitter. Another important input is the well known nigrostriatal dopaminergic system which originates from the pars compacta of the substantia nigra (SNpc). There has been a long debate as to whether dopaminergic inputs exert an excitatory or inhibitory effect on target neurons in the striatum, but there is now general consensus that the main effect is inhibitory. The striatum receives additional inputs from a number of other structures including the intralaminar nuclei and centrum medianum of the thalamus and the dorsal raphe nuclei of the midbrain, but there is only limited information concerning the function of these pathways. 


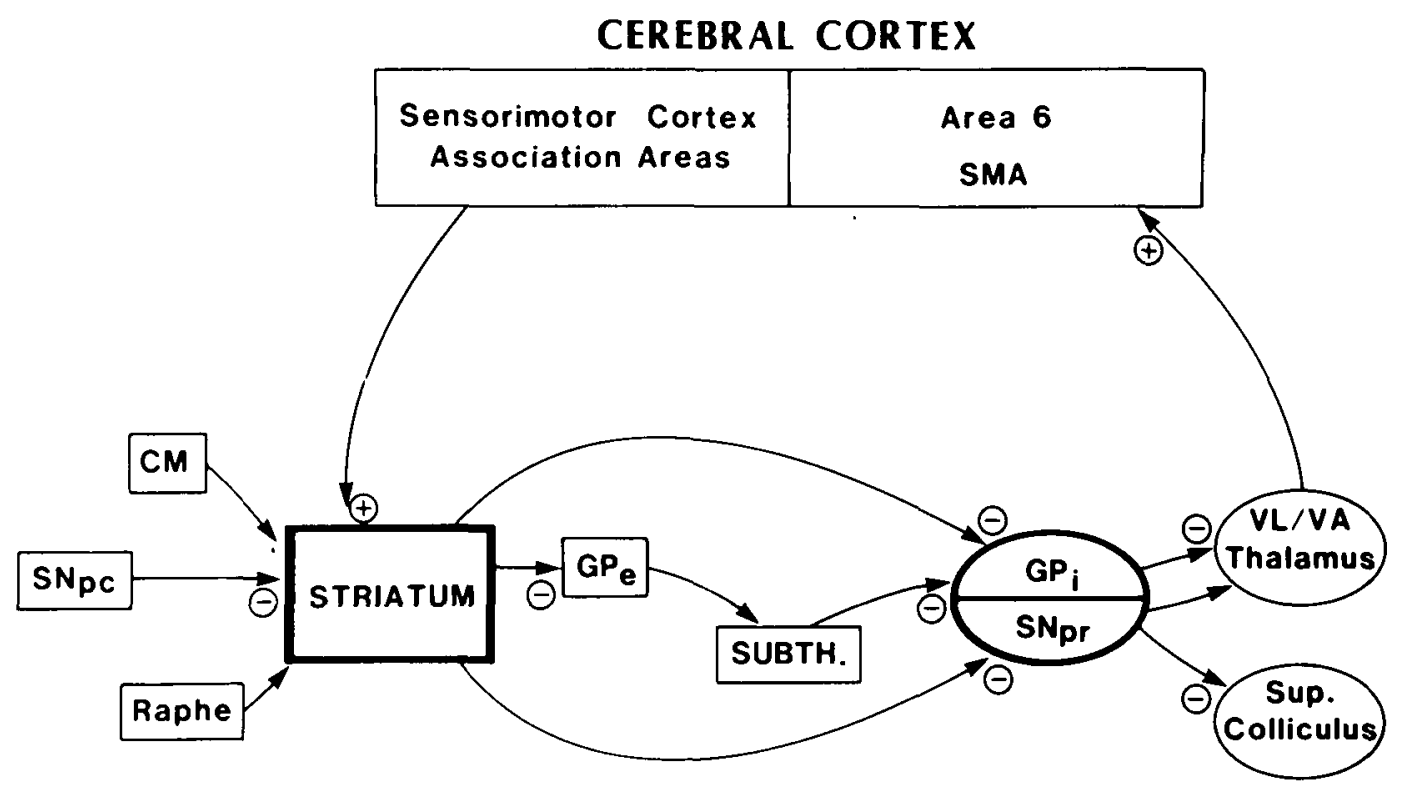

Figure I - Schematic diagram showing some of the anatomical connections of the basal ganglia. To present a simplified schema, several pathways have been omitted. Inputs to the striatum, the major receiving centre for the basal ganglia are shown on the left of the illustration. The main output nuclei and their efferent projections appear on the right.

Abbreviations used: Gpe and Gpi - external and internal segments of globus pallidus. SNpc and SNpr - substantia nigra pars compacta and pars reticulata $C M$ - centrum medianum, SMA - supplementary motor area, $\Theta=$ excitation, $\Theta=$ inhibition.

Until recently the two components of the striatum, the caudate and putamen, were considered to represent a single functional structure. However it is now known that projections to these two components originate from quite different regions of the cerebral cortex (Künzle, 1975). The caudate receives most of its inputs from regions considered to be "association" cortex, particularly the premotor regions of the frontal lobe and the posterior parietal areas. This anatomical organization is in keeping with other observations which suggest that the caudate is involved in certain types of cognitive activity and probably plays only a minor role in control of movement (Öberg and Divac, 1979). The putamen on the other hand is an integral part of the motor system and most of its cortical inputs originate from sensorimotor cortex (Areas 3, 1, 2, 4 and 6).

As shown in Figure 1, the striatum sends projections to both the external and internal segment of the globus pallidus (Gpe and Gpi) and also to the pars reticulata of the substantia nigra (SNpr). The Gpi and SNpr are considered to be the major relay stations for outputs from the basal ganglia. Neurons in Gpi and $\mathrm{SNpr}$ have many anatomical and physiological similarities, and it has been proposed that these areas really represent one structure which happens to have become separated during development by part of the internal capsule (Delong and Georgopoulos, 1979).

Projections from Gpi are directed mainly to the VL/VA complex of the thalamus. These fibers form synaptic connections with thalamic neurons which project back to cerebral cortex. The output from the basal ganglia is directed mainly to premotor cortex (Area 6) and the supplementary motor area (SMA) rather than to primary motor cortex (Area 4). This is another area where the organization of the basal ganglia differs from that of the cerebellum. Cerebellar output also passes through VL/VA but is directed mainly to Area 4. Although the output projections from the basal ganglia and cerebellum are in close proximity in the thalamus, they remain entirely separate and there is no convergence of information from these areas on to single thalamic neurons (Tracey et al., 1980).

The Gpi also sends projections to several other structures. These include the centrum medianum, the lateral habenula (providing a potential link between basal ganglia output and the limbic system), and a small nucleus in the mesencephalon known as nucleus tegmenti pedunculopontinus.

Studies by Delong and Georgopoulos (1979) have revealed a well defined somatotopic organization of movement related neurons in Gpi and SNpr. In Gpi neuronal activity is correlated with movements of the limbs, whereas in SNpr, the majority of cells which fire in association with specific movements do so with movements of head and neck structures. In this regard, it is interesting to note that one of the major targets for efferent projections from $\mathrm{SNpr}$ is the superior colliculus.

Although it has been recognized for a long time that lesions in or near the subthalamic nucleus cause hemiballismus of the contralateral extremities, this small structure has been largely ignored by physiologists. However, there has recently been renewed interest in the subthalamic nucleus and it is now believed that it may play a very important role in regulating basal ganglia output. It receives major inputs from Gpe but in addition there are extensive somatotopically organized projections from the motor cortex. The outputs from subthalamic nucleus are directed to $\mathrm{Gpi}$ and $\mathrm{SNpr}$. It is believed that these efferent pathways exert an inhibitory effect on their target neurons. Therefore, the subthalamic nucleus is in an unique position to modulate a large portion of the output coming from the basal ganglia.

What has been presented so far is of course a very incomplete and oversimplified picture. Figure 1 suggests that one could predict the result of increased or decreased activity in one component of the basal ganglia by merely summating excitatory and inhibitory effects and calculating the ultimate effect on cortical neurons. For example, damage to the nigrostriatal 
dopaminergic system removes a normal inhibitory influence on striatal neurons. As a result there could be increased activity in the inhibitory pathways to Gpi. This could result in decreased activity in the pathway from Gpi to VL/VA. It is believed that this is also an inhibitory pathway, so it is conceivable that the ultimate effect of loss of dopaminergic input to the striatum could be increased activity in thalamocortical projections. It is tempting to speculate that the resulting excitation of cortical neurons might be responsible for some of the features of parkinsonism which we associate with increased motor output.

However, block diagrams such as Figure 1 fail to indicate the complexity of internal wiring within each of the individual structures in the system. For example, although some of the afferent projections to the striatum act directly on striatal output neurons, many of the inputs from both cerebral cortex and $\mathrm{SNpc}$ appear to form synapses with cholinergic interneurons in the striatum. Furthermore, there is evidence for presynaptic receptors in the striatum and it has been proposed that the two major afferent systems projecting to the striatum are reciprocally linked at the presynaptic level as well as postsynaptically (Nieoullon et al., 1983). Thus, dopaminergic inputs from SNpc could influence activity of striatal neurons by direct inhibition and also by presynaptic inhibition of the excitatory inputs from cortex. It is obvious that much more complete wiring diagrams than that shown in Figure 1 are required before we can fully appreciate the role of the various components of the basal ganglia in control of normal movement. Recognizing that our knowledge of the circuitry is still far from complete, let us now consider some of the classical approaches which have been used by neurophysiologists in an attempt to understand the normal function of the basal ganglia. These include selective lesioning experiments, observations of the effects of electrical stimulation, and correlation of activity of single neurons with specific movements.

\section{Effects of Selective Lesions in the Basal Ganglia}

There is an extensive literature on the effects of electrolytic lesions involving selected regions of the basal ganglia, but the results have been controversial and difficult to interpret. (See Delong and Georgopoulos, 1981 for a detailed review). Perhaps the most surprising observation is that in many areas of the basal ganglia lesions produce very little motor deficit. In many cases, it has been necessary to make either very large lesions or bilateral lesions before obvious abnormalities appear. The most consistent result has been the production of contralateral hemiballismus with lesions of the subthalamic nucleus, but even here the results are not entirely specific and lesions in this area may also produce chorea and athetosis (Carpenter et al., 1950).

Unilateral lesions of $\mathrm{Gpi}$, which presumably interrupt much of the output from the basal ganglia, have no apparent effect on untrained animals. Bilateral lesions of $\mathrm{Gpi}$, unless they are very large, produce only mild transient hypokinesia. Similarly, unilateral lesions of the substantia nigra produce little or no motor deficit, although bilateral lesions in monkeys do produce hypokinesia, fixed postures, and some of the other features of parkinsonism. It is interesting to note that in monkeys lesions in this region do not produce tremor unless they include some of the cerebellar connections in the ventral tegmentum (Poirier, 1971). However, tremor has been observed in some patients with parkinsonism resulting from intoxication with 1-methyl- 4-phenyl-1,2,3,6-tetrahydropyridine (MPTP), a substance which appears to produce selective destruction of dopamineric neurons in SNpc (see papers by Langston and Burns, this volume). As mentioned previously, the caudate does not seem to play a major role in motor function and neither unilateral or bilateral caudate lesions produce any consistent motor deficit in experimental animals.

Studies which utilize electrolytic lesions are difficult to interpret because of the uncertainty in determining whether the effects are due to damage to neurons at the site of the lesion or to axons passing through the area. For this reason, some investigators have used other methods to produce selective dysfunction. One of these methods is localized cooling which blocks synaptic transmission without interfering with axons passing through the region. Unilateral cooling of the globus pallidus in monkeys results in increased dependence on visual feedback for limb movements, a tendency for the limbs to assume a flexed posture, and failure to achieve a correct balance between agonist and antagonist muscles (Hore and Villis, 1980). Some of these abnormalities have also been observed in parkinsonian patients (Cooke and Brown, 1979).

Another approach has been to use chemical toxins to produce damage to selected populations of neurons. The widest experience has been with 6-hydroxydopamine which produces extensive destruction of dopaminergic neurons. Chemical lesions of this type in rats result in spontaneous turning or circling. For the first 24 to 48 hours, circling is toward the side contralateral to the lesion. After 48 hours, there is consistent deviation of the head and trunk toward the side of the lesion, reduction in spontaneous behaviour, some degree of adipsia and aphagia and apparent sensory neglect of the contralateral side (Jungberg and Ungerstedt, 1976). In monkeys, unilateral 6-hydroxydopamine lesions also produce ipsilateral circling as well as bradykinesia and in some cases severe torticollis. It is tempting to speculate that these effects result from specific damage to the nigrostriatal dopaminergic neurons, but it is quite likely that 6-hydroxydopamine is much less selective than this in its effects and also damages other dopamineric neurons including those projecting to the limbic system.

\section{Electrical Stimulation of Basal Ganglia Structures}

In an attempt to understand the role of the basal ganglia in motor control, many investigators have studied the effects of electrical stimulation of specific sites in the basal ganglia in various animal species. Although electrical stimulation does have a number of different effects on motor behaviour, the results of these experiments are even more difficult to interpret than the lesion studies. Some of the abnormalities which have been observed with electrical stimulation include contraversive head turning and circling, flexion movements of the contralateral limbs, abrupt arrest of motor behaviour, licking, chewing, or swallowing movements, and modification of cortically induced movements. In some cases, the same phenomena have been observed with stimulation at several different sites in the basal ganglia. Stimulation at the same site may produce variable results depending on the strength and pattern which is used. Therefore, while the results of electrical stimulation support the concept that the basal ganglia are involved in control of movement, no clear picture has emerged from stimulation studies to help us understand the specific functions of various components of the basal ganglia. 


\section{Recordings from Single Nerve Cells in the Basal Ganglia}

During the past two decades, the development of techniques for chronic recording of discharge patterns of single neurons in monkeys trained to perform specific movements has added a new dimension to the investigation of basal ganglia physiology.

\section{Spontaneous Firing Patterns}

Spontaneous activity of neurons is quite different in various regions of the basal ganglia. Many neurons in the striatum appear to have no spontaneous activity and those which are active fire at relatively low rates. By contrast, neurons in the globus pallidus have characteristic high frequency bursts of spontaneous activity (Delong, 1971). In Gpe the bursts are relatively short while in $\mathrm{Gpi}$ and also in $\mathrm{SNpr}$, there are more sustained high frequency discharges (Figure 2). Cells in the pars compacta of substantia nigra have relatively low spontaneous firing rates, around 10 per second. In the subthalamic nucleus, spontaneous discharges occur at rates around 25 per second and there is some tendency for bursting to occur.

\section{Correlation of Single Unit Discharges With Specific Movements}

Most of these studies have focused on the major output centres of the basal ganglia, the Gpi and SNpr (Delong and Georgopoulos, 1979; Iansek and Porter, 1980). It has been observed that approximately $50 \%$ of identified neurons in these structures fire in relation to discrete movements of the limbs or of the orofacial structures. At least half of these neurons show a directional preference, that is they fire when movement of the

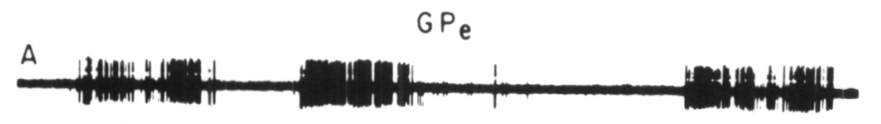

B
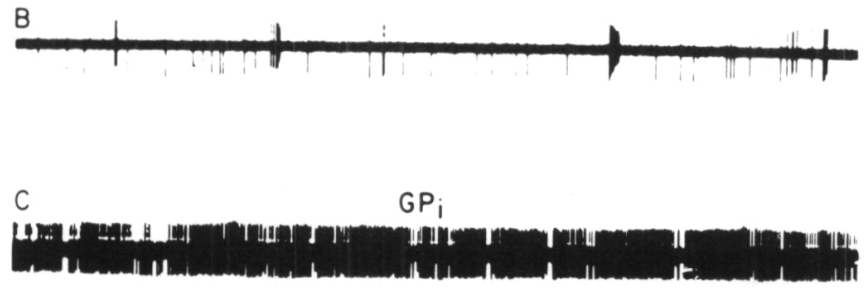

D BORDER

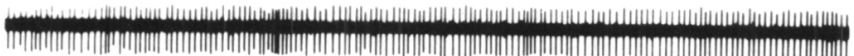

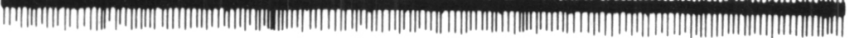

E

anch

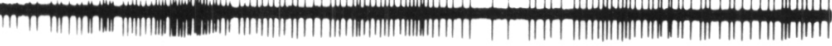
$500 \mathrm{msec}$

Figure 2 - Characteristicfiring patterns of basal ganglia neurons in a monkey at rest. $A$ and $B:$ two types of high frequency bursting seen in neurons in the external globus pallidus, $C$ : sustained high frequency discharge from a neuron in internal globus pallidus, $D$ : lower frequency sustained discharge from a neuron in the border zone between the two pallidal segments, and E: similar discharge from a neuron in substantia innominata from Delong, 1971, with permission). joint occurs in one direction but not when it occurs in the opposite direction. About $30 \%$ of neurons which fire in association with movement do so with movements of either the ipsilateral or contralateral extremities. Movements of proximal and distal muscles seem to be equally represented.

These single unit studies have confirmed that there is a well organized somatotopic organization. Figure 3 shows an example of a neuron in $\mathrm{Gpe}$ which demonstrates a clear association with pushing or pulling movements of the arm. The same neuron does not show any consistent increase in its firing rate in relation to similar movements of the leg. As mentioned previously, neurons which are associated with movements of the leg or arm are located for the most part in Gpi whereas neurons which fire in association with movement of the orofacial structures are located in SNpr.

Despite the fact that many neurons in these regions modulate their firing patterns in association with specific movements, the actual temporal relationship is variable. Some neurons fire in advance of the movement while others do not increase their firing rate until after the movement has commenced. Basal ganglia neurons do not seem to have the same tight temporal coupling to movement onset as has been observed with cells in the cerebellum or motor cortex.

In the putamen, the temporal relationship to movement is even less well established. Some cells appear to fire in relation to environmental stimuli which are going to trigger a movement, and the increase in firing rate may occur as long as 500-1000 msec. ahead of the actual movement, suggesting that these units are involved in some way in setting up or preparing for movement. Delong (1973) has observed that some units in the putamen fire preferentially in relation to slow ramp movements and not during more rapid movements of a ballistic type.

\section{Response to Peripheral Stimuli}

Many of the neurons in the basal ganglia which fire in relation to movement cannot be driven by any type of sensory input. Again, in contrast to the cerebellum, basal ganglia neurons show no consistent response to stimulation of cutaneous receptors. Some cells do respond to passive manipulation of the limbs which activates muscle stretch receptors and other deep structures

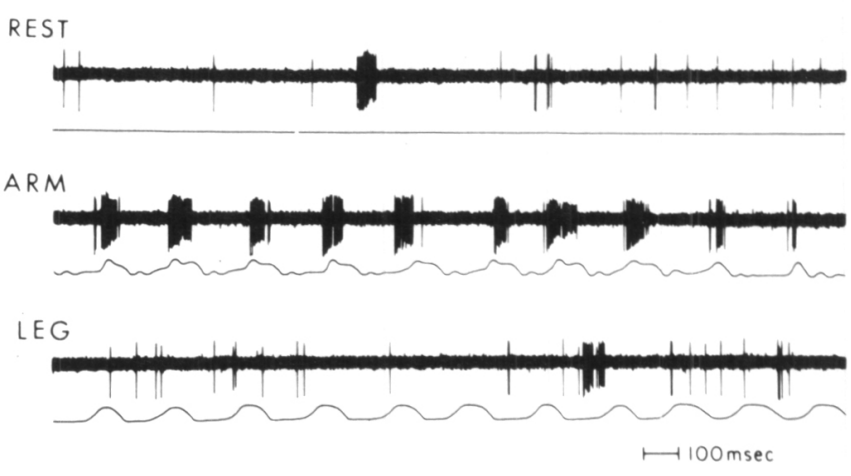

Figure 3 - Recording from a bursting neuron in the external globus pallidus which shows a clear relationship to pushing and pulling movements of the arm. No consistent firing is seen in association with similar movements of the leg (from Delong, 1971, with permission). 
in the extremities. However, these responses are relatively weak in comparison to what occurs during active volitional movement.

\section{Conclusions}

In this brief review, I have attempted to highlight a few representative examples selected from the very large number of physiological studies which have been done on the basal ganglia. Although considerable progress has been made, in my view, we are still a long way from a complete understanding of the role which the basal ganglia play in the control of normal movement and posture. The lesioning, stimulating, and recording experiments in animals have provided us with a large number of semi-isolated fragments of information, but we are still lacking the unifying thread required to tie all these pieces together into a meaningful hypothesis of movement.

It is interesting that our knowledge of what happens when some areas of the basal ganglia fail to function far exceeds our knowledge of what these structures actually do during normal movement. Observations on patients with Parkinson's disease and other movement disorders have lead to several hypotheses. For example, Marsden (1982) concludes that under normal conditions, the basal ganglia are responsible for "the automatic execution of learned motor plans". Penney and Young (1983) propose that the role of the striatum in movement is "to select which behaviours will be carried out and to suppress unwanted ones". Chorea and other involuntary movements are considered examples of unwanted movements. A detailed analysis of the motor deficits of Parkinson's disease can be found in the papers by Marsden and Tatton elsewhere in this volume.

Although observations on parkinsonian patients have contributed substantially to our understanding of the function of the basal ganglia, they have also raised a number of other questions which still require answers. Degeneration of neurons in SNpc and the resulting deficiency of striatal dopamine leads to major disturbances of movement. But what is the role of the nigrostriatal system during normal movement? Physiological studies have failed to establish any correlation between the discharge patterns of neurons in SNpc and any type of specific movement. It has been proposed that the dopaminergic input in some way modulates or facilitates activity in other systems within the striatum, but much more work is required before this role is fully understood.

The results of stereotaxic surgery on parkinsonian patients, as well as some of the lesioning experiments in animals, create an interesting paradox. If the basal ganglia are essential for normal movement, and there seems to be little argument concerning this, why is it that lesions which presumably interrupt a large portion of the neuronal output from the basal ganglia, result in little or no disturbance of voluntary movement?
The basal ganglia have always provided a common ground for meetings of basic investigators and clinical neurologists. Hopefully, the resulting interaction and the combined efforts of these two groups will provide answers to many of the questions concerning the function of this most interesting area of the central nervous system.

\section{REFERENCES}

Carpenter MB, Whittier JR, Mettler FA (1950) Analysis of choreoid hyperkinesia in the rhesus monkey. Surgical and pharmacological analysis of hyperkinesia resulting from lesions of the subthalamic nucleus of Luys. J. Comp. Neurol. 92:293-331.

Cook JD, Brown JD (1979) Increased dependence on visual information for arm movement in patients with Parkinson's disease. In The Extra Pyramidal System and Its Disorders, edited by LJ Poirier, TL Sourkes, PJ Bedard. Raven Press, New York, pp. 185-189.

Delong MR (1971) Activity of pallidal neurons during movement. J. Neurophysiol. 34:414-427.

Delong MR (1973) Putamen: activity of single units during slow and rapid arm movements. Science 179:1240-1242.

Delong MR, Georgopoulos AP (1979) Motor function of the basal ganglia as revealed by studies of single cell activity in the behaving primate. Adv. Neurol. 24:131-140.

Delong MR, Georgopoulos AP (1981) Motor functions of the basal ganglia. In Handbook of Physiol. Section I, Vol. II. Motor Control, edited by VB Brooks, Amer. Physiol. Society, pp. 1017-1061.

Hore J, Vilis T (1980) Arm movement performance during reversible basal ganglia lesions in the monkey. Exp. Brain Res. 39:217-228.

Iansek R, Porter R (1980) The monkey globus pallidus: neuronal discharge properties in relation to movement. J. Physiol. (Lond.) 301:439-455.

Jungberg T1, Ungerstedt U (1976) Sensory inattention produced by 6-hydroxydopamine induced degeneration of ascending dopamine neurons. Exp. Neurol. 53:585-600.

Kitai ST (1981) Electrophysiology of the corpus striatum and brain stem integrating system. In Handbook of Physiol. Section I, Vol. II. Motor Control, edited by VB Brooks, Amer. Physiol. Society, pp. 997-1015.

Künzle H (1975) Bilateral projections from precentral motor cortex to the putamen and other parts of the basal ganglia: an autoradiographic study in Macaca fascicularis. Brain Res. 88:195-209.

Marsden CD (1982) The mysterious motor function of the basal ganglia: the Robert Wartenberg lecture. Neurology 32:514-539.

Nieoullon A, Kerkerian L, Dusticier N (1983) Presynaptic controls in the neostriatum: reciprocal interactions between the nigro-strial dopaminergic neurons and the cortico-striatal glutamatergic pathway. In Neural Coding of Motor Performance, edited by J. Massion et al., Springer-Verlag, Berlin, pp. 54-65.

Öberg RGE, Divac I (1979) "Cognitive" functions of the neostriatum. In The Neostriatum, edited by I Divac, RGE Öberg. Pergamon Press, Oxford, pp. 291-313.

Penney JB Jr, Young AB (1983) Speculations on the functional anatomy of basal ganglia disorders. Ann. Rev. Neurosci. 6:73-94.

Poirier LJ (1971) The development of animal models for studies in Parkinson's disease. In Recent Advances in Parkinson's Disease, edited by FH McDowell, CH Markham, FA Davis, Philadelphia, pp. 83-117.

Tracey DJ, Asanuma C, Jones EG, Porter R (1980) Thalamic relay to motor cortex: afferent pathways from brain stem, cerebellum and spinal cord in monkeys. J. Neurophysiol. 44:532-544. 